\title{
THE $q$-DEFORMED CONVOLUTIONS: EXAMPLES AND APPLICATIONS TO MOMENT PROBLEM
}

\author{
ANNA KULA
}

\begin{abstract}
The paper provides examples of the $q$-convolution and the $(p, q)$-convolution, which put some light on how complicated these operations are. They show that the $q$-convolution of two Dirac delta's need not be compactly supported and that the $(1,1)$-convolution of two Dirac delta's can be absolutely continuous. A (possibly not unique) measure corresponding to a moment sequence from the limit theorem for the $(p, q)$-convolution is described.
\end{abstract}

Mathematics subject classification (2010): Primary: 28D05, 44A60. Secondary: 05A30, 42A85, 46L53.

Keywords and phrases: convolution, positive definiteness, indeterminate moment problems.

The paper is concentrated on two objects for which we use the common name " $q$-deformed convolutions". These are: the $q$-convolution, defined by Carnovale and Koornwinder [5], and the $(p, q)$-convolution, defined by Ricard and the author [9], $p$ and $q$ being two positive parameter.

The $q$-deformed convolutions both originates from the non-commutative probability and braided algebras, but they can also be interpreted as operations on sequences, involving the classical $q$-calculus. With this approach, as proved in [9], they turn out to be associative and commutative, and preserve a kind of strong positivity. By the latter we mean that they tranform the set of the $q$-moment sequences (moment sequences with "weights" depending on the parameter $q$ ) corresponding to measures on positive real half-line into itself. In the same paper, the (central) limit theorem for the $(p, q)$ convolution was established.

Although the $q$-convolution and $(p, q)$-convolution have already been studied, examples of them were unavailable untill now, and the aim of this paper is to fill this gap. After presenting the two convolutions and related notions (Section 1.), we describe four examples of the $q$-deformed convolutions (Section 2.). They put some light on how complicated operations the $q$-convolution and the $(p, q)$-convolution could be. In particular, we show that the $q$-convolution of two Dirac delta's need not be compactly supported and that the $(1,1)$-convolution of two Dirac delta's gives the (shifted) arcsine law. We also find a measure corresponding to moments from cental limit theorem for the $(p, q)$-convolution. Moreover, we show how the properties of the $q$-deformed convolutions help solving moment problem in some special cases. 


\section{Notation}

Given a (positive Borel) measure $\mu$ supported on the real-line $\mathbb{R}$, the moments of $\mu$ are defined by

$$
m_{n}(\mu)=\int t^{n} \mu(d t), \quad n \in \mathbb{N} .
$$

The set of all such measures which have all moments finite will be denoted by $\mathscr{P}_{f m}$. Similarily, the set of measures supported on the non-negative half-line $[0,+\infty)$ with all moments finite will be denoted by $\mathscr{P}_{f m}^{+}$. Moment sequences corresponding to measures from $\mathscr{P}_{f m}^{+}$are often called Stieltjes moment sequences.

For a measure $\mu \in \mathscr{P}_{\text {fm }}^{+}$and $a \geqslant 0, T_{a}$ denotes the dilation $\mu \mapsto T_{a} \mu$, defined for a measurable set $A \subset[0,+\infty)$ by the formula $\left(T_{a} \mu\right)(A)=\mu(A / a)$, when $a>0$, and $T_{0} \mu=\delta_{0}$. As usual, $\delta_{a}$ denotes the point mass measure concentrated at $a$.

Let us also recall that, by the Schur's Lemma, the pointwise product of two moment sequences is again a moment sequence. If, moreover, both sequences correspond to measures supported on $[0,+\infty)$, then the resulting sequence also shares this property. The corresponding transformation of measures is the multiplicative convolution, denoted by $\circ$. It can be defined for two measures $\mu, v$ as the image of the product measure $\mu \otimes v$ under the mapping $(x, y) \mapsto x y$. It follows that $\delta_{a} \circ \delta_{b}=\delta_{a b}$. Moreover, for measures $\mu, v \in \mathscr{P}_{f m}^{+}$we have

$$
m_{n}(\mu \circ v)=m_{n}(\mu) \cdot m_{n}(v), \quad n \in \mathbb{N} .
$$

Throughout all the paper, $p$ and $q$ are two positive parameters. Moreover, $\mathbb{N}=$ $\{0,1,2, \ldots\}$ is the set of non-negative integers. Unless otherwise stated, all sequences appearing below are indexed by $\mathbb{N}$. We also denote as usual (cf. [6])

$$
\begin{aligned}
& (a ; q)_{n}=\prod_{k=0}^{n-1}\left(1-q^{k} a\right), \quad(a ; q)_{\infty}=\lim _{n \rightarrow+\infty}(a ; q)_{n}, \\
& {[n]_{q}=\frac{1-q^{n}}{1-q}, \quad[n]_{q} !=\frac{(q ; q)_{n}}{(1-q)^{n}}, \quad\left[\begin{array}{l}
n \\
k
\end{array}\right]_{q}=\frac{[n]_{q} !}{[k]_{q} ![n-k]_{q} !} .}
\end{aligned}
$$

We shall need the $q$-binomial theorem ([6]):

$$
\sum_{k=0}^{+\infty} \frac{(a ; q)_{k}}{(q ; q)_{k}} z^{k}=\frac{(a z ; q)_{\infty}}{(z ; q)_{\infty}}, \quad|z|<1, \quad a \in \mathbb{C}
$$

and the formula

$$
\frac{(z ; q)_{\infty}}{\left(z q^{n} ; q\right)_{\infty}}=(z ; q)_{n}, \quad n \in \mathbb{N}
$$

\section{1. $q$-moment sequences and deformed convolutions}

DEFINITION 1. A sequence $\left(\mu_{n}\right)_{n}$ is called a $q$-moment sequence if there exists a (positive Borel) measure $\mu$ on $\mathbb{R}$ such that

$$
\mu_{n}=\mu_{n}^{(q)}=q^{\frac{n(n-1)}{2}} \int_{\mathbb{R}} t^{n} d \mu(t), \quad n \in \mathbb{N} .
$$


Note that if $q=1$, what we get is the standard notion of moment sequences. Since the parameter $q$ is fixed, we simplify the notation by omitting the superscript $(q)$ in $\mu_{n}^{(q)}$. The only problem which can appear is when considering moments and $q$-moments of a given measure. To avoid this, we will always denote by $\mu_{n}$ the $q$-moments of a measure $\mu$ wheras for the standard moments of $\mu$ we reserve the notation $m_{n}(\mu)$. In particular, $\mu_{n}^{(1)}=m_{n}(\mu)$ for $n \in \mathbb{N}$.

We will denote by $\mathscr{M}_{q}^{+}$the set of all $q$-moment sequences $\left(\mu_{n}\right)_{n}$ corresponding to measures from $\mathscr{P}_{f m}^{+}$. In a special case $q=1, \mathscr{M}_{1}^{+}$is the set of Stieltjes moment sequences. A nice description of $\mathscr{M}_{q}^{+}$for $q>0$ presented below contains the $q$ deformed version of the Stieltjes theorem (see [8] for details). In this paper, we will often use the equivalence $(1) \Leftrightarrow(3)$ from the following.

PROPOSITION 1.1. For a sequence $\left(\mu_{n}\right)_{n}$ with $\mu_{0}=1$ the following conditions are equivalent:

1. $\left(\mu_{n}\right)_{n} \in \mathscr{M}_{q}^{+}$, i.e. $\left(\mu_{n}\right)_{n}$ is a q-moment sequence corresponding to a probability measure $\mu$ on $[0,+\infty)$,

2. both sequences $\left(\mu_{n}\right)_{n}$ and $\left(\mu_{n+1}\right)_{n}$ are $q$-positive definite, that is, for all $n \in \mathbb{N}$ and all scalars $\alpha_{1}, \ldots, \alpha_{n} \in \mathbb{C}$ we have

$$
\sum_{i, j=0}^{n} q^{-i j} \alpha_{i} \bar{\alpha}_{j} \mu_{i+j} \geqslant 0 \text { and } \sum_{i, j=0}^{n} q^{-i j} \alpha_{i} \bar{\alpha}_{j} \mu_{i+j+1} \geqslant 0 .
$$

3. $\left(q^{-\frac{n(n-1)}{2}} \mu_{n}\right)_{n}$ is a moment sequence corresponding to a probability measure $\mu$ on $[0,+\infty)$.

From the papers [5] and [9], we derive the following definitions:

Definition 2. Let $\left(\mu_{n}\right)_{n}$ and $\left(v_{n}\right)_{n}$ belong to $\mathscr{M}_{q}^{+}$.We shall call:

1. the $q$-convolution of $\left(\mu_{n}\right)_{n}$ and $\left(v_{n}\right)_{n}$ the sequence given by the formula

$$
\left(\mu \star_{q} v\right)_{n}=\sum_{k=0}^{n}\left[\begin{array}{l}
n \\
k
\end{array}\right]_{q} \mu_{k} v_{n-k}, \quad n \in \mathbb{N}
$$

2. the $(p, q)$-convolution of $\left(\mu_{n}\right)_{n}$ and $\left(v_{n}\right)_{n}$ the sequence given by the formula

$$
\left(\mu \star_{p, q} v\right)_{n}=\sum_{k=0}^{n}\left(\frac{q}{p}\right)^{k(n-k)}\left[\begin{array}{l}
n \\
k
\end{array}\right]_{p}^{2} \mu_{k} v_{n-k}, \quad n \in \mathbb{N} .
$$

Two crucial facts concerning the behaviour of the $q$-convolution and $(p, q)$-convolution are Propositions 3.3 and 4.3 from [9], which we recall here.

PROPOSITION 1.2.

1. If $0<q<1$, then the $q$-convolution preserves the sequences from $\mathscr{M}_{q}^{+}$in the sense that the $q$-convolution of two sequences from $\mathscr{M}_{q}^{+}$belongs to $\mathscr{M}_{q}^{+}$. 
2. If $p, q>0$, then the $(p, q)$-convolution preserves the sequences from $\mathscr{M}_{q}^{+}$in the sense that the $(p, q)$-convolution of two sequences from $\mathscr{M}_{q}^{+}$belongs to $\mathscr{M}_{q}^{+}$.

In both cases, it is to be checked directly that the operation is associative and commutative. Moreover, the $(p, q)$-convolution is symmetric with respect to $p \leftrightarrow p^{-1}$. Easy examples show that the convolutions are not (inner) operations on the set of $q$ moments sequences corresponding to measures on $\mathbb{R}$ (see [9] for details).

Observe also that the 1-convolution coincides with the classical convolution of sequences, so the $q$-convolution can be called a $q$-deformation of the classical convolution. This is not the case with the $(p, q)$-convolution. Moreover, the $q$-convolution is not a special case of the $(p, q)$-convolution, but for $p=q$ we have

$$
\left(\mu \star_{q, q} v\right)_{n}=\sum_{k=0}^{n}\left[\begin{array}{l}
n \\
k
\end{array}\right]_{q}^{2} \mu_{k} v_{n-k},
$$

which differs from the $q$-convolution only by the second power of the $q$-binomial coefficient.

The motivation for both the $q$-convolution and the $(p, q)$-convolution comes from the non-commutative probability (in particular, braided algebras, cf. [9]) and that is why the $q$-moments fits better to describe this operations. It turns out however that, thanks to the characterisation of $\mathscr{M}_{q}^{+}$(Proposition 1.1), we are able to reveal their action on the classical moment sequences.

We start with the $q$-convolution. Let us take two sequences $\left(m_{n}(\mu)\right)_{n},\left(m_{n}(v)\right)_{n}$ from $\mathscr{M}_{1}^{+}$. Then, obviously,

$$
\mu_{n}=q^{\frac{n(n-1)}{2}} m_{n}(\mu), \quad v_{n}=q^{\frac{n(n-1)}{2}} m_{n}(v) \quad \text { for } n \in \mathbb{N}
$$

belong to $\mathscr{M}_{q}^{+}$and thus

$$
\begin{aligned}
\left(\mu \star_{q} v\right)_{n} & =\sum_{k=0}^{n}\left[\begin{array}{l}
n \\
k
\end{array}\right]_{q} \mu_{k} v_{n-k}=\sum_{k=0}^{n}\left[\begin{array}{l}
n \\
k
\end{array}\right]_{q} q^{\frac{k(k-1)}{2}+\frac{(n-k)(n-k-1)}{2}} m_{k}(\mu) m_{n-k}(v) \\
& =q^{\frac{n(n-1)}{2}} \sum_{k=0}^{n}\left[\begin{array}{l}
n \\
k
\end{array}\right]_{q} q^{-k(n-k)} m_{k}(\mu) m_{n-k}(v) .
\end{aligned}
$$

According to Proposition 1.2, the resulting sequence belongs to $\mathscr{M}_{q}^{+}$and by Proposition 1.1, $\left(q^{-\frac{n(n-1)}{2}}\left(\mu \star_{q} v\right)_{n}\right)_{n}$ belongs to $\mathscr{M}_{1}^{+}$. Thus there is a measure $\eta \in \mathscr{P}_{\text {fm }}^{+}$such that

$$
m_{n}(\eta)=\sum_{k=0}^{n}\left[\begin{array}{l}
n \\
k
\end{array}\right]_{q} q^{-k(n-k)} m_{k}(\mu) m_{n-k}(v) .
$$

This formula shows how the $q$-convolution transform two sequences from $\mathscr{M}_{1}^{+}$into a sequence from $\mathscr{M}_{1}^{+}$.

When we consider the $(p, q)$-convolution acting on moment sequences (instead of $q$-moment sequences), the parameter $q$ disappears. Indeed, for $\left(m_{n}(\mu)\right)_{n},\left(m_{n}(v)\right)_{n}$ 
from $\mathscr{M}_{1}^{+}$and $\left(\mu_{n}\right)_{n},\left(v_{n}\right)_{n} \in \mathscr{M}_{q}^{+}$as in (7), we have

$$
\begin{aligned}
\left(\mu \star_{p, q} v\right)_{n} & =\sum_{k=0}^{n}\left(\frac{q}{p}\right)^{k(n-k)}\left[\begin{array}{l}
n \\
k
\end{array}\right]_{p}^{2} \mu_{k} v_{n-k} \\
& =\sum_{k=0}^{n}\left[\begin{array}{l}
n \\
k
\end{array}\right]_{p}^{2} q^{\frac{n(n-1)}{2}} p^{-k(n-k)} m_{k}(\mu) m_{n-k}(v) \\
& =q^{\frac{n(n-1)}{2}} \sum_{k=0}^{n}\left[\begin{array}{l}
n \\
k
\end{array}\right]_{p}^{2} p^{-k(n-k)} m_{k}(\mu) m_{n-k}(v) .
\end{aligned}
$$

This means that the mapping

$$
\mathscr{M}_{1}^{+} \times \mathscr{M}_{1}^{+} \ni\left(\left(m_{n}(\mu)\right)_{n},\left(m_{n}(v)\right)_{n}\right) \rightarrow\left(m_{n}(\eta)\right)_{n} \in \mathscr{M}_{1}^{+}
$$

where

$$
m_{n}(\eta)=\sum_{k=0}^{n}\left[\begin{array}{l}
n \\
k
\end{array}\right]_{p}^{2} p^{-k(n-k)} m_{k}(\mu) m_{n-k}(v)
$$

is a well defined operation on $\mathscr{M}_{1}^{+}$, which depends only on the parameter $p>0$. This is exactly the $(p, 1)$-convolution.

Here again a similarity between $q$ - and $(q, 1)$-convolution is evident.

\section{Applications of the $q$-deformed convolutions}

\subsection{Example of the $q$-convolution}

In this section we assume that $0<q<1$. For $a>0$ let

$$
G_{n}(a)=G_{n}(a ; q)=\sum_{k=0}^{n}\left[\begin{array}{l}
n \\
k
\end{array}\right]_{q} q^{-k(n-k)} a^{k} .
$$

Then $\left\{G_{n}(a)\right\}_{n}$ is a Stieltjes moment sequence (i.e. a moment sequence of a measure on $[0,+\infty)$ ). This fact was shown by Al-Salam and Carlitz in [1], the authors used hypergeometric series' techniques.

An alternative proof is a straithforward application of the definition of the $q$ convolution.

Proposition 2.1. The sequence $\left\{G_{n}(a)\right\}_{n}$ is a Stieltjes moment sequence and

$$
m_{n}\left(\delta_{a} \star_{q} \delta_{1}\right)=G_{n}(a) .
$$

Proof. For the proof, let us consider $\mu=\delta_{a}(a>0)$ and $v=\delta_{1}$ and calculate the $n^{\text {th }}$ moment of $\delta_{a} \star_{q} \delta_{1}$. For this, we use Eq. (7) and obtain

$$
m_{n}\left(\delta_{a} \star_{q} \delta_{1}\right)=\sum_{k=0}^{n}\left[\begin{array}{l}
n \\
k
\end{array}\right]_{q} q^{-k(n-k)} a^{k}=G_{n}(a) .
$$


Thus the moments of $\delta_{a} \star_{q} \delta_{1}$ are exactly $\left\{G_{n}(a)\right\}_{n}$.

On the other hand, $\delta_{a}$ and $\delta_{1}$ are measures on $[0,+\infty$ ) (we assumed $a>0$ ) and so the sequence $\left\{G_{n}(a)\right\}_{n}$ is in $\mathscr{M}_{1}^{+}$.

REMARKS. 1. Unfortunately, the $q$-convolution gives no informations on the measure which corresponds to the moment sequence $\left\{G_{n}(a)\right\}_{n}$. It can be found in [1] or [4] that the two measures

$$
\begin{aligned}
m_{a} & =(a q ; q)_{\infty} \sum_{k=0}^{+\infty} \frac{a^{k} q^{k^{2}}}{(a q ; q)_{k}(q ; q)_{k}} \delta_{q^{-k}}, \quad 0<a<\frac{1}{q} \\
\sigma_{a} & =\left(a^{-1} q ; q\right)_{\infty} \sum_{k=0}^{+\infty} \frac{a^{-k} q^{k^{2}}}{\left(a^{-1} q ; q\right)_{k}(q ; q)_{k}} \delta_{a q^{-k}}, \quad q<a
\end{aligned}
$$

have the moment sequence $\left\{G_{n}(a)\right\}_{n}$ and one should choose $m_{a}$ or $\sigma_{a}$ depending on the relation between $a$ and $q$.

2 . In this paper, the $q$-convolution is treated as operation on $q$-moment sequences, but it would be interesting to generalize them to operations on measures ${ }^{1}$. A way to make it would be firstly generalize the definition to measures with compact support. The example of $\delta_{a} \star_{q} \delta_{1}$ shows that such a direct generalization is hardly possible, since the $q$-convolution of two compactly supported measures need not be compactly supported (the support can be unbounded as $0<q<1$ ). The situation is even worse, since the sequence $\left\{G_{n}(a)\right\}_{n}$ is well known as an example of indeterminate moment sequence (for $q<a<\frac{1}{q}$ both measures $m_{a}$ and $\sigma_{a}$ correspond to it). This means that the $q$-convolution of two compactly supported measures (which are necessarily uniquely determined by their moments) may no longer be uniquely determined by moments.

\subsection{Positive definiteness of the sequence $\left(q^{-\left(\begin{array}{c}n \\ 2\end{array}\right)}(-a ; q)_{n}\right)_{n}$}

This sequence is another example of the $q$-convolution and so is a moment sequence of a measure on $[0,+\infty)$.

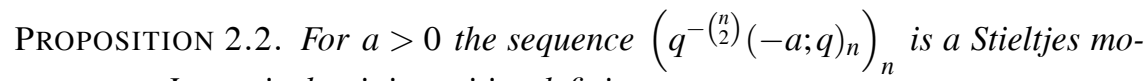
ment sequence. In particular, it is positive definite.

Proof. Let us fix $a>0$ and consider the $q$-moment sequence $\left(\mu_{n}\right)_{n}$ corresponding to the measure $\delta_{a}$, i.e. $\mu_{n}=q^{\left(\begin{array}{l}n \\ 2\end{array}\right)} a^{n}$ and the constant sequence $v_{n}=1=q^{\left(\begin{array}{l}n \\ 2\end{array}\right)} q^{-\left(\begin{array}{l}n \\ 2\end{array}\right)}$ for $n \in \mathbb{N}$. First we show that $\left(v_{n}\right)_{n}$ is in $\mathscr{M}_{q}^{+}$.

For this purpose, let us consider the log-normal distribution $\lambda_{\sigma}$ with parameter $\sigma>0$, which is the measure with the density on $[0,+\infty)$ given by

$$
d_{\sigma}(x)=\frac{1}{\sqrt{2 \pi \sigma^{2}}} x^{-1} \exp \left(-\frac{(\ln x)^{2}}{2 \sigma^{2}}\right) .
$$

\footnotetext{
${ }^{1}$ Some partial results can be found in [5].
} 
By a change of variable, one can show that ${ }^{2}$

$m_{n}\left(\lambda_{\sigma}\right)=\frac{1}{\sqrt{2 \pi \sigma^{2}}} \int_{0}^{+\infty} x^{n-1} \exp \left(-\frac{(\ln x)^{2}}{2 \sigma^{2}}\right) d x=\frac{1}{\sqrt{2 \pi \sigma^{2}}} \int_{0}^{+\infty} e^{n u+\frac{u^{2}}{2 \sigma^{2}}} d x=e^{\frac{1}{2} n^{2} \sigma^{2}}$.

So if $q \in(0,1)$ and we choose $\sigma^{2}=\ln \frac{1}{q}>0$, then $q^{\frac{1}{2}}=e^{-\frac{1}{2} \sigma^{2}}$ and $m_{n}\left(\lambda_{\ln 1 / q}\right)=q^{-\frac{n^{2}}{2}}$. Then, by the formula (1), we have

$$
q^{-\left(\begin{array}{c}
n \\
2
\end{array}\right)}=q^{-\frac{n^{2}}{2}} \cdot q^{\frac{n}{2}}=m_{n}\left(\lambda_{\ln 1 / q, s}\right) \cdot m_{n}\left(\delta_{\sqrt{q}}\right)=m_{n}\left(\lambda_{\ln 1 / q} \circ \delta_{\sqrt{q}}\right)=m_{n}\left(T_{\sqrt{q}} \lambda_{\ln 1 / q}\right) .
$$

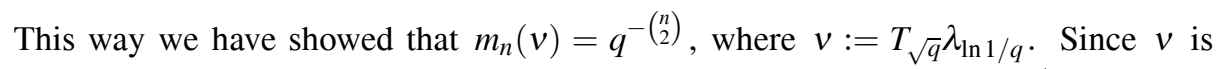
supported on $[0,+\infty)$, hence the constant sequence $(1)_{n}=\left(v_{n}\right)_{n}$ is in $\mathscr{M}_{q}^{+}$.

Now, we need the formula from the $q$-calculus

$$
(a ; q)_{n}=\sum_{k=0}^{n}\left[\begin{array}{l}
n \\
k
\end{array}\right]_{q} q^{\frac{k(k-1)}{2}}(-a)^{k}, \quad a \in \mathbb{C},
$$

(see [7] for the proof), from which we get for $a>0$

$$
(-a ; q)_{n}=\sum_{k=0}^{n}\left[\begin{array}{l}
n \\
k
\end{array}\right]_{q} \mu_{k} v_{n-k}=\left(\delta_{a} \star_{q} v\right)_{n},
$$

According to Proposition 1.2, the sequence $\left((-a ; q)_{n}\right)_{n}$ belongs to $\mathscr{M}_{q}^{+}$and so, by Proposition 1.1, $\left(q^{-\left(\begin{array}{c}n \\ 2\end{array}\right)}(-a ; q)_{n}\right)_{n}$ is in $\mathscr{M}_{1}^{+}$.

REMARKS. 1. We do not need to know the measure $\lambda_{\sqrt{q}}$ to check that the sequence $\left(q^{-\left(\begin{array}{c}n \\ 2\end{array}\right)}\right)_{n}$ is a Stieltjes moment sequence. By the classical Stieltjes Theorem, it is enough to verify that the sequence itself and the shifted sequence $\left(q^{-\left(\begin{array}{c}n+1 \\ 2\end{array}\right)}\right)_{n}$ are positive definite. But this follows from the fact that for $q \in(0,1)$ the matrix $\left[q^{-i j}\right]_{i, j=0}^{+\infty}$ is positive definite (see Lemma 15 in [10]).

2. It seems unclear to the author whether the sequence $\left((-a ; q)_{n}\right)_{n}$ is also positive definite. Instead, we can prove directly that $\left(\frac{1}{(-a ; q)_{n}}\right)_{n}$ belongs to $\mathscr{M}_{1}^{+}$. For this purpose, given $q \in(0,1)$ and $a>0$, let us define

$$
I_{q, a}=\frac{1}{(-a ; q)_{\infty}} \sum_{k=0}^{+\infty} \frac{q^{\left(\begin{array}{c}
k \\
2
\end{array}\right) a^{k}}}{(q ; q)_{k}} \delta_{q^{k}}
$$

This is a positive Borel measure on $[0,+\infty)$ and, by Eq. (2), its moments are

$$
m_{n}\left(I_{q, a}\right)=\frac{1}{(-a ; q)_{\infty}} \sum_{k=0}^{+\infty} \frac{q^{\left(\begin{array}{l}
k \\
2
\end{array}\right)\left(a q^{n}\right)^{k}}}{(q ; q)_{k}}=\frac{\left(-a q^{n} ; q\right)_{\infty}}{(-a ; q)_{\infty}}=\frac{1}{(-a ; q)_{n}} .
$$

\footnotetext{
${ }^{2}$ In fact, Stieltjes [11] showed that for all $s \in[-1,1]$ the distributions $\lambda_{\sigma, s}$ with densities

$$
d_{\sigma, s}(x)=\left(1+s \sin \left(\frac{2 \pi}{\sigma^{2}} \ln x\right)\right) d_{\sigma}(x)
$$

have the same moments $m_{n}\left(\lambda_{\sigma, s}\right)=e^{\frac{1}{2} n^{2} \sigma^{2}}$ (see also [2]).
} 


\subsection{Example of the $(1,1)$-convolution}

A basic tool in hypergeometric series' calculus is the $q$-binomial theorem (Eq. 2 ). In case of the $(p, q)$-convolution we lack this tool because of the second power of the $q$-binomial coefficient appearing in the formula. This makes the calulations of examples almost hopeless. The limit case as $p, q \rightarrow 1$ is a nice exception, which shows however that even the $(1,1)$-convolution is a complicated operation.

The $(1,1)$-convolution is the limit case of the $(p, q)$-convolution as $p, q \rightarrow 1$ and so it is defined as an operation on moment sequences via the formula

$$
m_{n}\left(\mu \star_{1,1} v\right)=\sum_{k=0}^{n}\left(\begin{array}{l}
n \\
k
\end{array}\right)^{2} m_{k}(\mu) m_{n-k}(v) .
$$

Proposition 2.3. For $a>0$ the measure $\delta_{a} \star_{1,1} \delta_{a}$ has the density function

$$
f(x)=\frac{1}{\pi} \frac{1}{\sqrt{x(4 a-x)}} \chi_{[0,4 a]}(x) .
$$

Proof. Let us recall that the following equality holds for central binomial coefficients:

$$
\left(\begin{array}{c}
2 n \\
n
\end{array}\right)=\sum_{k=0}^{n}\left(\begin{array}{l}
n \\
k
\end{array}\right)^{2} .
$$

For $a>0$ we have

$$
m_{n}\left(\delta_{a} \star_{1,1} \delta_{a}\right)=a^{n} \sum_{k=0}^{n}\left(\begin{array}{l}
n \\
k
\end{array}\right)^{2}=a^{n}\left(\begin{array}{c}
2 n \\
n
\end{array}\right) .
$$

We know however that $\left(\begin{array}{c}2 n \\ n\end{array}\right)$ is the $(2 n)^{\text {th }}$ moment of the dilated arcsine law, which has the density

$$
g(x)=\frac{1}{\pi} \frac{1}{\sqrt{4-x^{2}}} \chi_{[-2,2]} .
$$

This means that

$$
\begin{aligned}
a^{n}\left(\begin{array}{c}
2 n \\
n
\end{array}\right) & =\frac{a^{n}}{\pi} \int_{-2}^{2} \frac{x^{2 n}}{\sqrt{4-x^{2}}} d x=\frac{2}{\pi} \int_{0}^{2} \frac{\left(a x^{2}\right)^{n}}{\sqrt{4-x^{2}}} d x \\
& =\frac{1}{\pi} \int_{0}^{4 a} \frac{t^{n}}{\sqrt{t(4 a-t)}} d t .
\end{aligned}
$$

Thus $a^{n}\left(\begin{array}{c}2 n \\ n\end{array}\right)$ is the $n^{\text {th }}$ moment of the measure with the density

$$
f(x)=\frac{1}{\pi} \frac{1}{\sqrt{x(4 a-x)}} \chi_{[0,4 a]}(x) .
$$

REMARK. Since this measure is supported on the compact interval, the moment problem is determinate and the measure is unique. So, in contrast to the case of the $q$ convolution, this example does not exclude the possibility of generalizing the definition of the $(1,1)$-convolution to an operation on compactly supported measures. 


\subsection{Limit measure for the $(p, q)$-convolution}

In [9] the following result was proved.

THEOREM 2.4. (Limit Theorem for the $(p, q)$-convolution) Let $\left(\mu_{n}\right)_{n}$ be a q-moment sequence corresponding to a measure on $[0,+\infty)$ such that $\mu_{1}=1$. Then for every $n \in \mathbb{N}$ we have

$$
\lim _{K \rightarrow \infty}(T_{\frac{1}{\sqrt{K}}} \underbrace{\left(\mu \star_{p, q} \mu \star_{p, q} \ldots \star_{p, q} \mu\right)}_{K-\text { times }})_{n}=q^{\frac{n(n-1)}{2}} \frac{[n]_{p} ![n]_{p^{-1}} !}{n !} .
$$

Untill now, the measure related to this $q$-moments was not known. In this paper, we shall use the results of Berg from [3] to describe it as a multiplicative convolution of two measures. We briefly recall them. As in Berg's paper, we assume that $0<q<1$ and $0<p<1$.

We consider the measure

$$
R_{q}=(q ; q)_{\infty} \sum_{k=0}^{\infty} \frac{q^{k}}{(q ; q)_{k}} \delta_{q^{k}}
$$

which, by Eq.(2) and Eq.(3), has moments

$$
m_{n}\left(R_{q}\right)=(q ; q)_{\infty} \sum_{k=0}^{\infty} \frac{q^{(n+1) k}}{(q ; q)_{k}}=\frac{\left(q^{n+1} ; q\right)_{\infty}}{(q ; q)_{\infty}}=(q ; q)_{n} .
$$

We denote by $I_{q}$ the measure with the (non-negative) density function on $(0,+\infty)$

$$
i_{q}(x)=\frac{1}{(q ; q)_{\infty}} \sum_{k=0}^{\infty} \exp \left(-x q^{-k}\right) \frac{(-1)^{k} q^{\frac{k(k-1)}{2}}}{(q ; q)_{k}} \chi_{(0,+\infty)}(x)
$$

Then, by the $q$-binomial theorem (Eq. 2), the moments of $I_{q}$ are

$$
\begin{aligned}
m_{n}\left(I_{q}\right) & =\frac{1}{(q ; q)_{\infty}} \sum_{k=0}^{\infty} \frac{(-1)^{k} q^{\frac{k(k-1)}{2}}}{(q ; q)_{k}} \int_{0}^{+\infty} x^{n} e^{-x q^{-k}} d x \\
& =\frac{1}{(q ; q)_{\infty}} \sum_{k=0}^{\infty} \frac{(-1)^{k} q^{\frac{k(k-1)}{2}}}{(q ; q)_{k}} \int_{0}^{+\infty} q^{-k(n+1)} t^{n} e^{-t} d t \\
& =\frac{\left(q^{n+1} ; q\right)_{\infty}}{(q ; q)_{\infty}} \cdot n !=\frac{n !}{(q ; q)_{n}}
\end{aligned}
$$

Berg showed that $J_{q}:=\frac{1}{x \log \frac{1}{q}} d I_{q}(x)$ is also a probability measure. If by $\breve{J}_{q}$ we denote the image of $J_{q}$ under the reflection $x \rightarrow \frac{1}{x}$, then we have

$$
\check{J}_{q}=\frac{i_{q}\left(\frac{1}{x}\right) d x}{x \log \frac{1}{q}}
$$


and the moments of $\check{J}_{q}$ are

$$
\begin{aligned}
m_{n}\left(\check{J}_{q}\right) & =\frac{1}{\ln \frac{1}{q} \cdot(q ; q)_{\infty}} \sum_{k=0}^{\infty} \frac{(-1)^{k} q^{\frac{k(k-1)}{2}}}{(q ; q)_{k}} \int_{0}^{+\infty} x^{n-1} e^{-x^{-1} q^{-k}} d x \\
& =\frac{1}{\ln \frac{1}{q} \cdot(q ; q)_{\infty}} \sum_{k=0}^{\infty} \frac{(-1)^{k} q^{\frac{k(k-1)}{2}} q^{-k n}}{(q ; q)_{k}} \int_{0}^{+\infty} t^{-(n+1)} e^{-t} d x \\
& =\frac{\Gamma(-n)\left(q^{-n}, q\right)_{\infty}}{\ln \frac{1}{q} \cdot(q ; q)_{\infty}}=\frac{(q, q)_{n}}{n !} q^{-\frac{(n+1) n}{2}} .
\end{aligned}
$$

Above, $\Gamma(z)$ is the Gamma function and the last equality follows from the formula

$$
\Gamma(-n)\left(q^{-n}, q\right)_{\infty}=\frac{(q, q)_{n}(q ; q)_{\infty}}{n !} q^{-\frac{(n+1) n}{2}} \ln \left(\frac{1}{q}\right)
$$

which can be proved by induction (see [3] for an analytic proof).

We are interested in finding a measure $\lambda$ corresponding to the $q$-moment sequence $\lambda_{n}=q^{\frac{n(n-1)}{2}} \frac{[n]_{p} ![n]_{p}-1}{n !}$ or, equivalently by Proposition 1.1 , to the moment sequence $m_{n}(\lambda)=\frac{[n]_{p} ![n]_{p^{-1}} !}{n !}$. For this, let us observe that

$$
\begin{aligned}
\frac{[n]_{p} ![n]_{p^{-1}} !}{n !} & =p^{-\frac{n(n-1)}{2}}[n]_{p} ! \frac{[n]_{p} !}{n !} \\
& =\frac{p^{-\frac{n(n-1)}{2}}}{(1-p)^{2 n}}(p ; p)_{n} \frac{(p ; p)_{n}}{n !} \\
& =\frac{p^{n}}{(1-p)^{2 n}} m_{n}\left(R_{p}\right) m_{n}\left(\check{J}_{p}\right) \\
& =m_{n}\left(\delta_{p}\right) \cdot m_{n}\left(\delta_{\frac{1}{(1-p)^{2}}}\right) \cdot m_{n}\left(R_{p}\right) \cdot m_{n}\left(\check{J}_{p}\right)
\end{aligned}
$$

As stated in Eq. (1), the multiplication of moment sequences reflects the multiplicative convolution of measures, so the moment sequence in question corresponds to the measure

$$
\lambda \stackrel{\mathrm{m}}{=} \delta_{p} \circ \delta_{\frac{1}{(1-p)^{2}}} \circ R_{p} \circ \check{J}_{p}=\delta_{\frac{p}{(1-p)^{2}}} \circ R_{p} \circ \check{J}_{p}
$$

If we denote by

$$
\tilde{R}_{p}=\delta_{\frac{p}{(1-p)^{2}}} \circ R_{p}=(p ; p)_{\infty} \sum_{n=0}^{\infty} \frac{p^{n}}{(p ; p)_{n}} \delta_{\frac{p^{n+1}}{(1-p)^{2}}}
$$

then

$$
\lambda \stackrel{\mathrm{m}}{=} \tilde{R}_{p} \circ \check{J}_{p}
$$

which means that 
PROPOSITION 2.5. The measure corresponding to the limit moment sequence $\left(\frac{[n]_{p} ![n]_{p^{-1}} !}{n !}\right)_{n}$ is the multiplicative convolution of the measures $\tilde{R}_{q}$ and $\check{J}_{q}$.

REMARKS. 1. The fact that the sequence $\left(\frac{[n]_{p} ![n]_{p^{-1}} !}{n !}\right)_{n}$ is a Stieltjes moment sequence was proved in [9] (Collorary 6.3). It is worth noting that the formula (9) provides an alternative proof of this fact, since multiplicative convolution preserves measures on $[0,+\infty)$.

2. The determinancy of $\check{J}_{q}$ is not known (cf. [3]). In particular, the Carleman criterion gives no information about it, because the series

$$
\sum_{n} \frac{1}{\sqrt[2 n]{m_{n}\left(\check{J}_{q}\right)}}
$$

is convergent. Thus it is unknown if $\lambda$ is uniquely determined by its moments.

Acknowledgements. This research was partially supported by the Polish Ministry of Science's Grant NN 201364436.

\section{REFERENCES}

[1] W. A. Al-Salam, L. CARlitz, Some orthogonal q-polynomials, Math. Nachr. 30 (1965), 47-61.

[2] CH. BERG, On some indeterminate moment problems for measures on a geometric progression, Journal of Computational and Applied Mathematics 99 (1998), 67-75.

[3] CH. BERG, On a generalized Gamma convolution related to the $q$-calculus In: "Theory and Applications of Special Functions", Dev. Math. 13 (2005), 61-76.

[4] CH. Berg, G. VAlent, The Nevanlinna parametrization for some indeterminate Stieltjes moment problems associated with birth and death processes, Methods and Appl. Analysis, 1 (1994), 169-209.

[5] G. Carnovale, T. H. Koornwinder, A q-analogue of convolution on the line, Methods Appl. Anal., 7 (2000), 705-726.

[6] G. Gasper, M. Rahman, Basic Hypergeometric Series, Encyclopedia of Mathematics and its Applications, Vol. 96, Cambridge University Press, Cambridge 2004.

[7] T. H. KoORnwinder, Special functions and q-commuting variables, Fields Inst. Commun., 14 (1997), 127-166.

[8] A. KulA, A q-analogue of complete monotonicity, Colloq. Math., 111 (2008), 169-181.

[9] A. Kula, E. RiCARD, On a convolution for $q$-normal elements, Infin. Dimens. Anal. Quantum Probab. Relat. Top., 11 (2008), 565-588.

[10] S. Ôta, F. H. Szafraniec, q-positive definiteness and related topics, J. Math. Anal. Appl., 329 (2007), 987-997.

[11] T.-J. Stieltues, Recherches sur les fractions continues, Ann. Fac. Sci. Toulouse Math., 8 (1894), J1-J122; 9 (1895), A5-A47. 